\title{
Reorganização do processo de trabalho da Atenção Primária à Saúde durante o enfrentamento da pandemia da COVID-19: relato de experiência
}

\author{
Reorganización del proceso de trabajo de atención primaria de salud frente a la pandemia \\ de COVID-19: informe de experiencia
}

Reorganization of the primary health care work process in the north region of the country during the coaching of the pandemic COVID-19: experience report

Recebid o: 05 jul 2021

Revisado: 05 set 2021

Aceito: 14 set 2021

Autor de correspondência: Átila Augusto Cordeiro Pereira atilaacp@gmail.com

Como citar: Pereira ÁAC, Monteiro DS, Galvão SSC, Garcia LVF, Leal TF, Rosa JVM, Borges SCR. Reorganização do processo de trabalho da atenção primária à saúde durante o enfrentamento da pandemia da covid-19: relato de experiência J Manag Prim Health Care. 2021;13:e024. https://doi.org/10.14295/jmphc.v13. 1136

Conflito de interesses: Os autores declaram não haver nenhum interesse profissional ou pessoal que possa gerar conflito de interesses em relação a este manuscrito.

Copyright: Este é um artigo de acesso aberto, distribuído sob os termos da Licença Creative Commons (CC-BY-NC). Esta licença permite que outros distribuam, remixem, adaptem e criem a partir do seu trabalho, mesmo para fins comerciais, desde que the atribuam o devido crédito pela criação original.

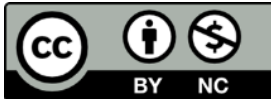

\author{
Átila Augusto Cordeiro PEREIRA ${ }^{(1)}$ \\ Diully Siqueira MONTEIRO ${ }^{(2)}$ \\ Sâmela Stefane Correa GALVÃO(1) \\ Layse Viana Figueiredo GARCIA ${ }^{(3)}$ \\ Tamilis Feitosa $\operatorname{LEAL}^{(1)}$ \\ João Victor Moura ROSA ${ }^{(4)}$ \\ Sâmia Cristine Rabelo BORGES ${ }^{(5)}$
}

\footnotetext{
(1) Universidade Federal do Pará - UFPA, Programa de Pós-graduação em Saúde, Ambiente e Sociedade na Amazônia - PPGSAS, Belém, PA, Brasil.

(2) Universidade do Estado do Pará - UEPA, Programa de Residência Multiprofissional em

Estratégia Saúde da Família, Belém, PA, Brasil.

(3) Secretaria Municipal de Saúde, Chaves, PA, Brasil.

(4) Universidade Federal do Pará - UFPA, Programa de Pós-graduação em Enfermagem - PPGENF, Belém, PA, Brasil.

(5) Secretaria Municipal de Saúde, Ananindeua, PA, Brasil.
}

\section{Resumo}

O artigo tem como objetivo descrever a experiência da reorganização do processo de trabalho na Atenção Primária à Saúde para o enfrentamento da COVID-19, no estado do Pará. Estudo qualitativo, do tipo relato de experiência, realizado no ano de 2021, a partir das experiências de profissionais de saúde de distintas regiões do estado. Ao longo da pandemia no estado a APS passou por reorganização do processo de trabalho buscando minimizar os efeitos da pandemia na região. Em resposta a este cenário destacam-se as medidas de distanciamento social, visitas domiciliares, combate a fake news, teleatendimento, reorganização da agenda de atendimento ao usuário, implantação de fluxos para atendimento de síndrome gripal, integração com a vigilância em saúde, somando um conjunto de ações que foram capazes de reduzir o contágio pela COVID-19 em municípios paraenses. Este processo de reorganização do processo de trabalho da APS enfrentou as dificuldades peculiares da região amazônica como o fator renda, rede de senviços de saúde, escolaridade, escassez de insumos, alta rotatividade de profissionais de saúde e barreiras geográficas. A reorganização das equipes de atenção primária foi importante para atender as demandas da população na medida em que garantiu acesso a serviços básicos de saúde. Persiste ainda a baixa disponibilidade de testes para identificação do novo coronavírus, falta de resolutividade no setor diagnóstico e laboratorial e a frágil integração da APS com a vigilância em saúde, o que tem proporcionado desafios para o rastreamento e subnotificação de casos na população paraense.

Descritores: Pandemias; COVID-19; Atenção Primária à Saúde; Fluxo de Trabalho; Sistema de Saúde Comunitária. 


\title{
Resumen
}

El artículo tiene como objetivo describir la experiencia de la reorganización del proceso de trabajo en Atención Primaria de Salud para enfrentar el COVID-19, en el estado de Pará. Estudio cualitativo, tipo informe de experiencia, realizado en 2021, a partir de experiencias de profesionales de la salud de diferentes regiones. del Estado. A lo largo de la pandemia en el estado, la APS experimentó una reorganización del proceso de trabajo, buscando minimizar los efectos de la pandemia en la región. En respuesta a este escenario, medidas de distanciamiento social, visitas domiciliarias, combate a fakenews, telecall, reorganización de la agenda de atención al usuario, implementación de flujos de atención tipo gripe, integración con la vigilancia de la salud, sumando un conjunto de acciones destacadas. capaz de reducir el contagio por COVID-19 en los municipios de Pará. Este proceso de reorganización del proceso de trabajo de la APS enfrentó las peculiares dificultades de la región amazónica, como el factor ingresos, la red de servicios de salud, la educación, la escasez de insumos, la alta rotación de profesionales de la salud y las barreras geográficas. La reorganización de los equipos de atención primaria fue importante para atender las demandas de la población, ya que garantizaba el acceso a los servicios básicos de salud. Todavía existe una baja disponibilidad de pruebas para identificar el nuevo coronavirus, la falta de resolución en el sector de diagnóstico y laboratorio, y la frágil integración de la APS con la vigilancia de la salud, lo que ha presentado desafíos para el seguimiento y subregistro de casos en la población de Pará.

Palabras-claves: Pandemias; COVID-19; Atención Primaria de Salud; Flujo de Trabajo; Servicios de Salud Comunitaria.

\begin{abstract}
The article aims to describe the experience of the reorganization of the work process in Primary Health Care to face COVID-19, in the state of Pará. Qualitative study, experience report type, carried out in 2021, from experiences of health professionals from different regions of the state. Throughout the pandemic in the state, the APS underwent a reorganization of the work process, seeking to minimize the effects of the pandemic in the region. In response to this scenario, measures of social distancing, home visits, combating fake news, teleservice, reorganization of the user service agenda, implementation of flu-like care flows, integration with health surveillance, adding a set stand out. of actions that were able to reduce the contagion by COVID-19 in Pará municipalities. This process of reorganization of the PHC work process faced the peculiar difficulties of the Amazon region, such as the income factor, health services network, education, scarcity of inputs, high turnover of health professionals and geographic barriers. The reorganization of primary care teams was important to meet the demands of the population as it guaranteed access to basic health services. There is still a low availability of tests to identify the new coronavirus, lack of resolution in the diagnostic and laboratory sector, and the fragile integration of $\mathrm{PHC}$ with health surveillance, which has provided challenges for the tracking and underreporting of cases in the Pará population.
\end{abstract}

Keywords: Pandemics; COVID-19; Primary Health Care; Workflow; Comunnity Health Services.

\section{Introdução}

No fim de 2019, o vírus denominado SARS-CoV-2 surge causando uma série de casos de pneumonia na cidade de Wuhan (China), doença denominada como COVID-19 e que em 2020 disseminou para os demais continentes, resultando na pandemia que fez milhares de mortos em todo o mundo. A pandemia pela doença do novo coronavírus (COVID-19) atingiu mais de 220 milhões de pessoas até o momento, e provocou a morte de mais de 4 milhões delas no mundo, segundo dados da Organização Mundial da Saúde - OMS. ${ }^{1}$

O conhecimento sobre o manejo clínico para o novo coronavírus vem passando por diversos estudos, mas sabe-se que o vírus tem alta transmissibilidade e provoca síndrome 
respiratória aguda que varia de casos leves - cerca de $80 \%$ - a casos muito graves com

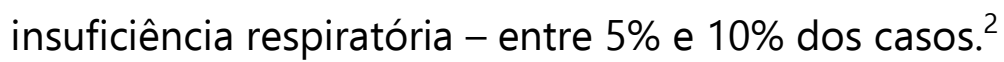

No Brasil, e em diversos países do mundo, a resposta sanitária tem sido centrada nos serviços hospitalares, com ações para a ampliação do número de leitos, especialmente, de unidades de tratamento intensivo e respiradores pulmonares. Sem retirar a importância da adequada estruturação da atenção especializada voltada aos casos mais graves da COVID-19, é preciso alertar que, no âmbito da Atenção Primária à Saúde - APS, muito pode e precisa ser feito. ${ }^{3}$

Segundo o Painel COVID-19, no Brasil até o dia 17 de setembro de 2021, foram registrados 21.034.610 casos confirmados da COVID-19 e 588.597 óbitos decorrentes dessa enfermidade no país, ocupando o ranking de $3^{\circ}$ país com mais casos confirmados. A Região Norte obteve 1.809 .756 de casos e 45.539 óbitos. Em relação ao estado do Pará, localizado na Região Norte, foram registrados 587.992 casos confirmados e 16.578 óbitos. $^{4-6}$

A infecção pelo COVID-19 na Região Norte do país torna-se ainda mais preocupante, visto que a região possui grandes iniquidades geográficas e sociais que se refletem em seus indicadores socioeconômicos. Ainda que nos últimos anos tenha ocorrido um aumento da cobertura de Equipes de Saúde da Família - eSF no Brasil, ${ }^{7}$ a Região Norte é a que apresenta, proporcionalmente, uma das menores coberturas de APS do país, possui o menor número de leitos de UTI, de médicos e de respiradores, recursos indispensáveis no tratamento e no combate a infecções respiratórias e complicações por COVID-19. ${ }^{8}$

As comunidades ribeirinhas do estado do Pará, como as da região do Marajó, trazem consigo peculiaridades diferentes dos demais municípios e região metropolitana. Nesse cenário de pandemia e de fragilidade da APS, as equipes de vigilância epidemiológica têm estado à frente na identificação dos casos suspeitos de COVID-19, estando muito mais presentes em campo e em alguns lugares assumindo isoladamente quase toda demanda daquele município. ${ }^{9-12}$

A dispersão territorial, o acesso geográfico e os serviços de saneamento e abastecimentos de água precários aumentam as condições de vulnerabilidade nas comunidades ribeirinhas do estado do Pará. O distanciamento entre os domicílios torna-se um fator de proteção durante a pandemia, evitando assim o aumento da contaminação pela COVID-19, por outro lado há de se considerar as condições de moradia da maior parte dessas comunidades compostas por muitas pessoas em poucos cômodos e distantes, muitas vezes, dos principais pontos de atenção à saúde. ${ }^{11,12}$ 
Com a evolução da transmissão comunitária se interiorizando no estado, intensificou-se a fiscalização de portos e de barreiras nos municípios paraenses. Neste momento o déficit histórico de recursos humanos e equipamentos nos órgãos de fiscalização do SUS, principalmente nos municípios marajoaras ganhou visibilidade. Faltavam meios de locomoção para as ações de vigilância em saúde e serviços de atenção secundária e terciária em algumas localidades e torna-se um obstáculo para o combate à pandemia e a execução das demais políticas de saúde. Estes órgãos encontraram grande dificuldade de atender as denúncias de descumprimento do decreto estadual e municipal para o isolamento social em portos e embarcações (voadeiras), principal transporte usado nestas comunidades. ${ }^{11,12}$

Mesmo com os estudos desenvolvidos sobre o novo coronavírus é importante conhecer como a pandemia vem atingindo as regiões e qual impacto no processo de trabalho da APS e no Sistema Único de Saúde - SUS. Este estudo tem por objetivo descrever a experiência da reorganização do processo de trabalho na Atenção Primária à Saúde para o enfrentamento da COVID-19, no Estado do Pará.

\section{Metodologia}

Estudo de relato de experiência desenvolvido por enfermeiras e enfermeiros que estiveram na qualidade de agente técnico da gestão e participaram da reorganização do processo de trabalho na APS para o enfrentamento da COVID-19, em Belém, Benevides e Chaves no estado do Pará, no período de março de 2020 a fevereiro de 2021.

O relato de experiência é uma ferramenta descritiva que apresenta um fato vivenciado, para contribuir de forma relevante para a atuação profissional e para a comunidade científica, trazendo considerações que proporcionem reflexões e embasamento teórico para outros pesquisadores. ${ }^{13}$

O Estado do Pará, está localizado na região Norte do Brasil e é composto por 144 municípios, dividido em 6 mesorregiões criadas pelo Instituto Brasileiro de Geografia e Estatística - IBGE: Baixo Amazonas constituído por 15 municípios, Marajó com 16, Metropolitana de Belém com 11, Nordeste Paraense formado por 49, Sudeste Paraense por 39 e Sudoeste Paraense com 14 municípios. $^{14}$

Para além da organização geográfica a fim de realizar o planejamento e avaliação em saúde, o Pará é dividido em treze regiões de saúde, subdivido em quatro macrorregiões de saúde como espaço regional ampliado para garantir a resolutividade das Redes de Atenção à Saúde - RAS. Nesse sentido, Belém e Benevides estão inclusos na 
ISSN 2179-6750

região de saúde Metropolitana I, já o município de Chaves está na Região Marajó I. Os cenários das vivências estão juntos na Macrorregião de Saúde I. ${ }^{15}$

Os municípios de Belém e Benevides estão estabelecidos na mesorregião Metropolitana de Belém e Chaves na mesorregião do Marajó. De acordo com a classificação do IBGE, quanto à tipologia, Belém e Benevides estão classificados como urbanos e Chaves como rural remoto. Possuem uma população estimada para 2021 de 1.506.420, 64.780 e 24.174 pessoas, respectivamente. ${ }^{15}$

Belém destaca-se com alta densidade demográfica representada por 1.315,26 $\mathrm{hab} / \mathrm{km}^{2}$ e Índice de Desenvolvimento Humano Municipal - IDHM de 0,746. Quanto ao seu território, possui 67,9\% de condições de esgotamento sanitário adequado. Diferente de Belém, o município de Benevides possui uma densidade demográfica de 274,99 hab/ $\mathrm{km}^{2}$, $17,4 \%$ do território possui esgotamento sanitário adequado, mas possui IDHM de 0,665, próximo ao de Belém. ${ }^{15}$ Por outro lado, o município de Chaves apresenta baixa densidade demográfica, 1,61 hab/ $\mathrm{km}^{2}$, IDHM de 0,453 e apenas 1,6\% do município encontra-se em condições de esgotamento sanitário adequado. Destaca-se pela proximidade com o município de Macapá, capital do Estado do Amapá, logo há uma importante influência quanto à circulação de pessoas e acesso aos serviços. ${ }^{15}$

Quanto ao panorama da APS no estado do Pará, no que diz respeito a cobertura populacional de Atenção Básica - AB, na competência dezembro de 2020 o estado possuía $64,49 \%$ de sua população coberta por $A B$ e $57,64 \%$ coberta por ESF, isto representa mais de 1.500 eSF no território paraense, dentre elas estão presentes também as equipes de Saúde da Família Ribeirinhas e Fluviais. ${ }^{16}$

\section{Panorama da APS em Belém antes da Pandemia da COVID-19}

De acordo com a última competência disponível no e-Gestor, de dezembro de 2020 o município de Belém possuía uma cobertura populacional de 39,97\% de Atenção Básica e 23,11\% de Estratégia de Saúde da Família - ESF, a menor cobertura de AB e ESF comparada às demais capitais do Brasil. Belém possui 29 Unidades Municipais de Saúde funcionando no modelo tradicional de Atenção Básica, que não trabalham na lógica de funcionamento de ESF. Até dezembro de 2020, possuía 100 eSF credenciadas pelo Ministério da Saúde - MS, destas 5 são de tipologia ribeirinha, divididos em 58 Unidades Básicas de Saúde - UBS, é comum ter até quatro eSF nas UBS. Possuía cadastrados no Cadastro Nacional de Estabelecimentos de Saúde - CNES 628 Agentes Comunitários de Saúde - ACS e 11 equipes do Núcleo Ampliado de Saúde da Família - NASF. ${ }^{16}$

As unidades possuem funcionamento no regime de 40 horas e organizam a agenda focada, predominantemente no atendimento materno infantil, caracterizando agenda em 
ISSN 2179-6750

torno de subpopulações definidas a exemplo, dia de consulta do pré-natal exclusivo em um turno. Quanto à rotina da sala de vacina, funciona no mesmo horário das unidades e é focalizada na administração de imunizantes com pouca instrumentalização para análise da cobertura do território. O acesso para triagem Neonatal é por demanda espontânea.

\section{Panorama da APS em Benevides antes da Pandemia da COVID-19}

Benevides possui $100 \%$ de cobertura populacional de AB/ESF, contabilizando 25 eSF, destas 1 de tipologia ribeirinha, localizada na zona rural do município, em 17 UBS. Possui cadastrados no CNES 135 ACS. Possui três equipes de NASF. ${ }^{16}$

As unidades funcionavam em regime de 40 horas semanais, aberta ao público das $8 \mathrm{~h}$ às $17 \mathrm{~h}$, mesmo horário de funcionamento das salas de vacina. Benevides por possuir cobertura completa de ACS realizava visitas domiciliares regularmente. A organização da agenda da APS em Benevides se baseava no cuidado materno infantil, cuidado à saúde do idoso, cuidado à saúde das pessoas com Doenças Crônicas Não Transmissíveis - DCNT, cuidados a pessoas com Doenças Infecciosas e Parasitárias - DIP, especialmente tuberculose e hanseníase, doenças negligenciadas hiperendêmicas na região.

\section{Panorama APS Chaves antes da Pandemia da COVID-19}

O município de Chaves localizado no extremo norte da ilha de Marajó, às margens do rio Amazonas, apresenta 78,1\% de sua população no meio rural, dispersa em fazendas, ilhas, margens de rios em pequenas comunidades e grandes fazendas e $21,9 \%$ no meio urbano. Tem uma zona rural extensa, impondo distância (às vezes de quilômetros) entre uma residência e outra, e pequenos aglomerados de casas nas regiões. Essa distribuição da população torna difícil o trabalho da equipe multiprofissional de saúde, tanto para o atendimento dos usuários, quanto para a vacinação de rotina, em decorrência da migração demográfica para acessibilidade aos serviços de saúde nos referidos municípios de fronteira com o estado do Amapá, incluindo ainda o município de Afuá (Pará), que atende uma demanda considerável de munícipes que procuram os seus serviços de saúde.

Chaves possui 58,19\% de cobertura populacional de AB/ESF, contabilizando 4 eSF, destas três de tipologia ribeirinha, localizadas às margens dos rios e uma na sede do município, possui uma Unidade Básica de Saúde Fluvial - UBSF ainda não credenciada pelo MS, que até o momento encontra-se ancorada no porto municipal. Possui cadastrados no CNES 70 ACS. Não possui Núcleo Ampliado de Saúde da Família - NASF. ${ }^{16}$ 
O presente estudo dispensa a aprovação do Comitê de Ética em Pesquisa por se tratar de relato de experiência, constituindo-se da descrição de atividades em serviço e utiliza informações de domínio público.

\section{Resultado e discussão}

A OMS recomenda que para suprimir a infecção pela COVID-19, em função da transmissão exponencial, é preciso utilizar de estratégias como o distanciamento social, testagem massiva e isolamento dos casos. ${ }^{17}$

No pico da pandemia, os testes do Ministério da Saúde chegaram com atraso e em quantidade insuficiente para toda população, contribuindo para as subnotificações dos casos iniciais. Os exames laboratoriais disponíveis direcionados para estados e municípios também foram limitados, o que levou muitas prefeituras do estado a realizarem compra emergencial de teste rápido. ${ }^{18}$ Atualmente os exames laboratoriais já são oferecidos pela rede privada, os testes rápidos podem ser encontrados na rede do SUS e a tão aguardada vacina contra COVID-19, embora já seja uma realidade mundial, no Brasil a velocidade de vacinação ainda é muito lenta. ${ }^{19}$

A partir disso, com a premissa de que a doença se trata de uma infecção respiratória, os estados, o Distrito Federal e municípios iniciam suas linhas de controle, prevenção e tratamento baseados nos planos para a pandemia de influenza e síndromes respiratórias, previstos no Guia de Vigilância Epidemiológica - GVE e manuais técnicos para enfrentamento de eventos epidemiológicos em massa, fundamentando a capacidade e experiência do SUS frente à resposta de combate ao novo coronavírus. ${ }^{20}$

Ainda que tenha ocorrido nos últimos anos um aumento $(35,8 \%)$ das pessoas moradoras em domicílios cadastrados em unidades de saúde da família no estado do Pará, evidenciando a aceleração da expansão da cobertura populacional da ESF, a média desta cobertura está abaixo da média nacional. ${ }^{7}$

No Pará, todos os municípios contam com eSF. Entretanto, existem importantes diferenças na cobertura, no acesso e na oferta de cuidados nas UBS, das diversas regiões do estado, em parte em função de mecanismos de gestão e das desigualdades sociais, com repercussões importantes no acesso e uso dos serviços de saúde. Também é encontrada uma correlação negativa entre a população dos municípios e a cobertura das equipes. Quanto menor o porte populacional, maior o percentual de cobertura de APS. ${ }^{7}$

Uma parcela significativa dos municípios paraenses é composta por população ribeirinha, de baixa renda e expectativa de vida, tem a subsistência voltada para atividade pesqueira e agrícola, especialmente do açaí, dispõem de serviços pouco articulados em 
ISSN 2179-6750

rede comprometendo o cuidado integral, falta de insumos e dificuldade de fixação de profissionais de saúde, alta rotatividade de gestores e as barreiras geográficas que afetam a oferta de serviços e a logística da subsistência cotidiana dessa população. ${ }^{11,12}$

No curso da Pandemia, na competência de maio de 2020, a Região Norte obteve um aumento na cobertura de APS e passou para $72,14 \%$, no entanto teve diminuição da modalidade ESF, com 62,82\%. O estado do Pará diminuiu a cobertura de APS para $64,23 \%$ sendo 57,44\% de ESF. Em Belém, a cobertura de APS representou 40,47\% sendo 22,19\% de ESF, Benevides se manteve com 100\% sendo exclusivo de ESF, e Chaves diminuiu para 29,09\%, também exclusiva de modalidade ESF. ${ }^{16}$

Atualmente existem 8 UBSF credenciadas no estado do Pará para atender a população que vive nas margens, destas 7 estão sendo pagas regularmente pelo MS nos seguintes municípios: Abaetetuba, Almeirim, Bagre, Breves, Curralinho, Melgaço e Santarém. $^{16}$

Neste cenário, tendo em vista que a maioria dos casos de infectados pela COVID-19 evolui com sintomas leves e moderados, a APS como porta de entrada do SUS, possui papel fundamental no manejo clínico dos casos leves e moderados e identificação precoce de casos graves que devem ser direcionados aos serviços especializados. ${ }^{2}$

Diante da infecção pelo novo coronavírus, no início dos primeiros casos no estado do Pará, a APS em conjunto com os canais de atendimento do estado e municípios, desempenhou atividades voltadas à orientação da população quanto à etiqueta respiratória, aos hábitos de higiene e cuidados domiciliares. ${ }^{9}$ No primeiro momento da pandemia, os municípios tiveram dificuldades em adquirir Equipamentos de Proteção Individual - EPI para os profissionais de saúde e equipamentos hospitalares como oxímetros, termômetros, álcool em gel, dentre outros, devido à escassez no mercado. ${ }^{21}$

A falta destes EPI foi uma realidade na saúde brasileira, a pandemia agravou, elevou a demanda e houve um salto nos preços destes produtos dificultando a aquisição em vários municípios. Nesta pandemia, a falta dos EPIs foi relatada em diversos serviços da rede de atenção à saúde no Brasil. ${ }^{22}$

O MS alertou para mudanças necessárias no processo de trabalho dos Agentes Comunitários de Saúde - ACS, como manter as visitas domiciliares, porém em áreas peridomiciliares, e enfatizou seu papel determinante na identificação precoce e acompanhamento dos casos suspeitos no território. A Região Norte que possui a terceira menor cobertura de eSF com 62,2\%, ficando atrás do Nordeste $(73,3 \%)$ e Sul $(67,6 \%)$, teve ainda uma redução nas visitas domiciliares em virtude do grande número de ACS afastados por força de decretos que recomendava afastamento das atividades laborais de trabalhadores com idade avançada, doenças crônicas e adoecimento pela COVID-19. ${ }^{7,9}$ 
$\mathrm{O}$ isolamento social deve ser incentivado por todos os profissionais da equipe, principalmente pelos ACS, mobilizando lideranças e recursos locais com ampla divulgação de informações e realização de medidas concretas. A literatura tem mostrado que os ACS são importantes aliados no enfrentamento de epidemias, especialmente no que diz respeito à conscientização da população e combate ao estigma relacionado à doença, o que realça o seu papel na difusão de informações corretas sobre a prevenção de COVID$19 .^{3,23}$

Com as medidas de isolamento social, amplamente divulgadas, o fluxo de atendimento da APS precisou ser adaptado para evitar aglomerações. Fez-se necessário a reorganização da agenda e rotina de atendimento nesses serviços, que adotaram algumas estratégias como:

\section{Estratégias adotadas}

Organização da agenda para os atendimentos das outras condições de saúde em bloco de horas;

Ampliação do horário de funcionamento de algumas unidades de saúde;

Ampliação do tempo de renovação de receitas a pacientes com doenças crônicas não transmissíveis (DCNT);

Atendimentos aos usuários que se enquadraram como prioridades após estratificação de risco e vulnerabilidade;

Adaptação de modo que as principais linhas de cuidado não tivessem descontinuidade como as gestantes, pessoas com diabetes e/ou hipertensão;

Fluxo diferenciado para os usuários com síndrome gripal;

Implantação do teleatendimento por alguns municípios;

Combate às fake news nas páginas oficiais de algumas gestões municipais.

Quadro 1. Estratégias adotadas pela Atenção Primária à Saúde - APS no contexto da pandemia de COVID-19

Fonte: Elaborado pelos autores (2021).

\section{Município de Belém}

No município de Belém o teleatendimento foi a principal ferramenta utilizada para monitoramento dos casos de COVID-19 dentro dos marcos da pandemia. O processo de reorganização do trabalho na APS na capital paraense sofreu o impacto do primeiro 
momento, mas em curto intervalo de tempo organizou treinamento em teleatendimento para funcionários da prefeitura, residentes e voluntários em um espaço dentro da secretaria de saúde, especificamente no Departamento de Vigilância em Saúde - DEVS. Por diversas vezes esta atividade colapsou com o avançar da pandemia, obrigando o gestor a realizar busca frequente por mais voluntários.

$\mathrm{Na}$ atenção clínica individual, utilizando modalidades de teleatendimento, profissionais da APS puderam orientar os casos suspeitos quanto ao isolamento e reconhecimento dos sinais de alerta; identificar pacientes que não podem ser cuidados no domicílio; monitorar estes casos suspeitos quanto à evolução clínica; realizar vídeo consultas para casos mais complexos e solicitar remoção para uma unidade hospitalar ao identificar sinais de agravamento. ${ }^{24}$

Apesar de ser a capital do Estado, Belém apresenta baixa cobertura de ESF, o município tentou realizar atendimento de usuários que se enquadraram como prioridades após estratificação dos riscos e vulnerabilidades assim como tentou evitar descontinuidade nas principais linhas do cuidado como gestantes, pessoas com diabetes e hipertensão, no entanto, se depararam com um absenteísmo nunca visto antes e várias equipes completamente desfalcadas, agravando a frágil cobertura de ESF do município.

Não houve no âmbito da APS de Belém a construção de duplo fluxo para evitar contatos com pessoas com sintomas gripais, tão pouco ampliação do horário de atendimento como em outros municípios. A população quando apresentava sintomas gripais moderados, se dirigia com muita frequência para as Unidades de Pronto Atendimento à Saúde - UPAS e para as Policlínicas do Governo do Estado.

O combate às notícias falsas, as fake news, foi um artifício utilizado por todos os municípios em seus canais oficiais, rádios e telejornais, haja vista o volume delas em um cenário ainda de descobertas e incertezas sobre a doença e sua cura ou tratamento.

\section{Município de Benevides}

O município de Benevides, localizado a $32 \mathrm{~km}$ de Belém, portanto integrando a Região Metropolitana da capital, teve experiências diferentes no contexto da pandemia de COVID-19. Por ser um município com $100 \%$ de cobertura de ESF, este foi mais exitoso no monitoramento dos casos e contou com apoio decisivo dos ACS e ACE.

Diante dos primeiros casos no município, o Departamento de Atenção Básica DEAS determinou a ampliação do tempo para renovação de receitas de pacientes com doenças crônicas não transmissíveis por um período de três meses, intermediado pelo ACS. Em seguida definiu duplo fluxo para atendimento de pacientes na APS, nas policlínicas e unidades com mais de uma ESF, de modo que um lado se garantia o 
atendimento aos usuários com síndrome gripal e de outro os atendimentos de outras clínicas.

O atendimento a outras condições de saúde seguiu à seguinte priorização: atendimento por demanda espontânea nos casos de gestantes, pessoas com diabetes e hipertensão e horários agendados para atender outras condições clínicas em bloco de horas ao longo do dia.

Benevides enfrentou dificuldades para realizar teleatendimento por falta de estrutura e recursos adequados e por conta dessa peculiaridade, uma ESF localizada ao lado da única UPA do município, teve suas dois ESF realocadas para o território adjacente e em seu lugar o município instalou um ponto de apoio da Vigilância Epidemiológica do município. Essa adaptação visava ser suporte de informações para a população, centralizava as testagens para os casos mais graves, devido à escassez de testes, e dava mais celeridade na identificação dos casos que chegavam à UPA e precisavam ser encaminhados para Belém com urgência.

No mês seguinte ao início da pandemia, o município realizou novos contratos para o DEVS e iniciou a descentralização das testagens em todo território conforme abastecimento dos testes na localidade e ampliou o serviço para regime de plantão de 24 horas. Deste modo os testes e os profissionais da vigilância puderam alcançar as áreas rurais, ribeirinhas e mais remotas do município de noite e aos finais de semana.

\section{Município de Chaves}

O município de Chaves, por sua vez, realizou monitoramento dos pacientes e dos contatos dos casos por teleatendimento (ligação e via whatsapp). Os casos eram monitorados pela equipe da ESF e os contatos monitorados pela vigilância epidemiológica. Os atendimentos de síndrome gripal foram realizados no anexo do hospital criado para atendimento de COVID-19.

Já os pacientes graves eram encaminhados para Macapá via hidroviária por ambulancha devido sua proximidade com a capital Amapaense.

A localização geográfica do município enfrentou uma barreira relacionada especialmente à logística para atendimento de saúde da população e para as atividades de fiscalização realizadas pela vigilância em saúde, pois a baixa estrutura como falta de meios de locomoção (embarcações) foram fatores limitantes.

\section{Considerações finais}

Foi evidente o impacto que a pandemia trouxe para a rede de atenção primária, ainda que gestores tenham tentado reorganizar o processo de trabalho da APS para sentir 
este efeito de forma branda. A cobertura da APS se apresentou como determinante para o maior controle da doença entre os municípios assim como a integração com a vigilância em saúde. Municípios que melhor conseguiram integrar as ações da APS com as da vigilância em saúde foram mais exitosos no enfrentamento da pandemia.

Diversas ações da APS foram prejudicadas como as ações de saúde bucal que passou a atender somente casos de urgências na maioria dos municípios e o atendimento de saúde mental sofreram descontinuidades irreparáveis nesta região.

O impacto também reverberou nas atividades coletivas de Educação em Saúde. As Academias da Saúde tiveram suas atividades suspensas, a fim de evitar contato entre os usuários que frequentam os grupos de atividades físicas e dança. Assim como os grupos de tabagistas, gestantes, adolescentes, idosos também tiveram suas atividades suspensas. $^{24}$

O Programa Nacional de Imunização - PNI que já vinha apresentando dificuldades em atingir suas metas, sentiu também os efeitos da crise sanitária. Apenas 51,20\% da população alvo recebeu a dose da vacina contra a Influenza $(\mathrm{H} 1 \mathrm{~N} 1)$ e a campanha contra o sarampo tem enfrentado grandes desafios que tem dificultado o combate à circulação da doença em muitas localidades. Alguns municípios utilizaram estratégias para evitar aglomeração nas unidades de saúde, em especial de idosos e pessoas com comorbidades, como vacinação em área externa, vacinação extramuros e em domicílio, principalmente para a terceira idade e pessoas acamadas, conforme orientações do $\mathrm{MS}^{25}$

No entanto, com a pandemia da COVID-19 e a semelhança dos sintomas com o vírus Influenza, a procura pela vacina da gripe foi maior que nos anos anteriores. Essa situação não garantiu aumento de doses de imunobiológicos para os municípios paraenses, o que ocasionou insatisfação da população e transtorno em alguns municípios para conseguir garantir o acesso ao atendimento e prevenção, contra H1N1, da população alvo. A pandemia coincidiu com o inverno amazônico, período de sazonalidade das doenças gripais na população da região.

Neste cenário, Savassi et al. ${ }^{26}$ explicam os impactos da COVID-19 na APS e que podem ser divididos em três ondas. A $1^{\circ}$ onda acontece por meio da morbimortalidade da COVID-19, o que já vem ocorrendo desde o início da pandemia, com o pico no estado do Pará entre os meses de abril e maio de 2020. A $2^{\circ}$ onda se caracteriza pela restrição de recursos para problemas agudos e crônicos assistidos na APS, o que impacta em novas práticas para contemplar as sequelas causadas pela doença $\mathrm{A} 3^{\circ}$ onda se caracteriza pelos impactos em saúde mental, que irão provocar os serviços a se reorganizarem e a possível sobrecarga do mesmo, tendo em vista, a carência de uma rede psicossocial resolutiva. 
ISSN 2179-6750

Estudos internacionais corroboram a importância do modelo de saúde baseado no território e com a forte atuação do ACS no combate a pandemia. ${ }^{23}$ Logo, as medidas tomadas até o presente momento para reorganizar a APS, devem se intensificar e exigir esforços de equipes para que os atendimentos de casos de síndrome gripal sejam incluídos em protocolos assistenciais e linhas de cuidados já utilizados na APS. ${ }^{27}$

Nos municípios ribeirinhos do estado, há um destaque para a necessidade de equipamentos de proteção individual nas UBS, falta de resolutividade no setor diagnóstico laboratorial e a baixa fixação de profissionais, o que tem proporcionado desafios para o rastreamento de casos de COVID-19. Esta população é parcialmente contemplada na Política Nacional de Atenção Básica - PNAB de 2017 carecendo da incorporação e conexão com a Política Nacional de Saúde Integral da População do campo, floresta e das águas. ${ }^{28}$

Recomenda-se a realização de novos estudos sobre a temática com o intuito de problematizar as disparidades de recursos destinados para a Região Norte e as medidas que ainda poderiam ser implementadas a fim de ampliar a resolutividade da APS e reduzir radicalmente as disparidades regionais e consequentemente o padrão de saúde dos brasileiros moradores da Amazônia.

\section{Contribuição autoral}

Todos os autores participaram da concepção, delineamento, planejamento, análise, interpretação, redação e revisão crítica do trabalho. Todos aprovaram a versão final encaminhada.

\section{Referências}

1. World Health Organization. Coronavirus disease (COVID-19) pandemic: outbreak situation. Geneva: WHO; 2020.

2. Ministério da Saúde (BR). Protocolo de manejo clínico do coronavírus (COVID-19) na atenção primária à saúde. Brasília, DF: Secretaria de Atenção Primária à Saúde; maio 2020 [citado 10 fev 2021]. Disponível em: https://portaldeboaspraticas.iff.fiocruz.br/wpcontent/uploads/2020/05/20200504_ProtocoloManejo_ver09.pdf

3. Medina MG, Giovanella L, Bousquat A, Mendonça MHM, Aquino R; Comitê Gestor da Atenção Primária à Saúde da Abrasco. Atenção primária à saúde em tempos de COVID19: o que fazer?. Cad Saude Publica. 2020;36(8):e00149720. https://doi.org/10.1590/0102-311X00149720. 
4. Johns Hopkins University. Dashboard by the center for systems science and engineering (CSSE) covid-19 [Website]. Baltimore (MD): JHU; 2020 [citado 19 set 2021]. Disponível em: https://coronavirus.jhu.edu/map.html

5. Coronavírus (covid-19). Google notícias [Website]. [local desconhecido]: Google; [c2020; citado 19 set 2021]. Disponível em: https://news.google.com/covid19/map?hl=ptBR\&mid $=\% 2 F m \% 2 F 0 d 0 k 4 \& g l=B R \& c e i d=B R \% 3 A p t-419$

6. Secretaria de Saúde do Estado do Pará. Coronavírus no Pará. Belém: SESPA; 2020 [citado 15 mar 2021]. Disponível em: http://www.saude.pa.gov.br/coronavirus/

7. Giovanella L, Bousquat A, Schenkman S, Almeida PF, Sardinha LMV, Vieira MLFP. The family health strategy coverage in Brazil: what reveal the 2013 and 2019 national health surveys. Cien Saude Colet. 2021;26(suppl 1):2543-56. https://doi.org/10.1590/141381232021266.1 .43952020 .

8. Mendonça FD, Rocha SS, Pinheiro DLP, Oliveira SV. Região norte do Brasil e a pandemia de covid-19: análise socioeconômica e epidemiológica. J Health NPEPS. 2020;5(1):20-37. https://doi.org/10.30681/252610104535.

9. Vale EP, Rodrigues GM, Costa DP, Queiroz JM, Lima DG, Medeiros LPF, et al. Reorganização da rede de atenção à saúde para o enfrentamento da covid-19 no município de Canaã dos Carajás, Pará. APS Rev. 2020;2(2):83-90. https://doi.org/10.14295/aps.v2i2.101.

10. Santos J. Populações ribeirinhas e educação do campo: análise das diretrizes educacionais do município de Belém-PA, no período de 2005-2012 [dissertação]. Belém (PA): Universidade Federal do Pará; 2014.

11. Morais CG, Gomes IS, Lima JG, Costa TLS. Abaré I: reflexões sobre formação multiprofissional e saúde ribeirinha na atenção primária à saúde. J Manag Prim Healh Care. 2021;13:e011. https://doi.org/10.14295/jmphc.v13.1043.

12. Almeida ER, Brandão CC, Matielo E, Santana MA, Ugarte AO, Costa TS. Atenção básica à saúde: avanços e desafios no contexto amazônico. In: Schweickardt JC, El Kadri MR, Lima RTS, organizadores. Atenção básica na região Amazônica: saberes e práticas para o fortalecimento do SUS. Porto Alegre: Rede Unida; 2019. p. 15-50. (Série saúde e Amazônia; 8). https://doi.org/10.18310/9788554329266. 
13. Lopes MVO. Sobre estudos de casos e relatos de experiências [Editorial]. Rev Rene [Internet]. 2012 [citado 11 set 2021];13(4):1. Disponível em: http://periodicos.ufc.br/rene/article/view/4019

14. Governo do Pará. Subdivisões [Internet]. Belém: SIC.PA; [data desconhecida; citado 20 set 2021]. Disponível em: https://www.pa.gov.br/pagina/54/subdivisoes

15. Instituto Brasileiro de Geografia e Estatística. Benevides: panorama [Website]. Brasília, DF: IBGE; c2017 [citado 20 set 2021]. Disponível em: https://cidades.ibge.gov.br/brasil/pa/benevides/panorama

16. Ministério da Saúde (BR). e-Gestor Atenção Básica [Website]. Brasília, DF: MS; 2021 [citado 20 set 2021]. Disponível em: https://egestorab.saude.gov.br/paginas/acessoPublico/relatorios/relHistoricoCobertura.x html

17. Bittencourt RJ. Testagem de rastreio e busca ativa de infectados assintomáticos pelo SARS-COV-2: a visão do planejamento em saúde pública. Comun Cien Saude. 2020:31:816. https://doi.org/10.51723/ccs.v31iSuppl\%201.649.

18. Tribunal de Contas dos Municípios do Estado do Pará. Mural de licitações: consulta pública [Internet]. Belém: TCM-PA; c2017 [citado 20 set 2021]. Disponível em: https://www.tcm.pa.gov.br/mural-delicitacoes/licitacoes/ficha/QT6FleORVSz00d\#documentos

19. Centro Brasileiro de Estudos de Saúde. Frente pela vida cobra mais doses, mais recursos e adequada coordenação das ações para acelerar a vacinação contra a covid-19 [Internet]. [local desconhecido]: Cebes; 12 fev 2021 [citado 12 fev 2021]. Disponível em: http://cebes.org.br/2021/02/frente-pela-vida-mais-doses-mais-recursos-maiscoordenacao-contra-covid/

20. Ministério da Saúde (BR). Infecção humana pelo novo coronavírus. Bol Epidemiol. fev 2020 [citado 16 fev 2021];(2):1-23. Disponível em: https://portalarquivos2.saude.gov.br/images/pdf/2020/fevereiro/13/Boletimepidemiologico-COEcorona-SVS-13fev20.pdf

21. Teixeira CFS, Soares CM, Souza EA, Lisboa ES, Pinto ICM, Andrade LR, et al. The health of healthcare professionals coping with the covid-19 pandemic. Cien Saude Colet. 2020;25(9):3465-74. https://doi.org/10.1590/1413-81232020259.19562020. 
22. Cabral ERM, Bonfada D, Melo MC, Cesar ID, Oliveira REM, Bastos TF, et al. Contribuições e desafios da atenção primária à saúde frente à pandemia de covid-19. InterAmerican J Med Health. 2020;3:e202003012. https://doi.org/10.31005/iajmh.v3i0.87.

23. Haines A, Barros EF, Berlin A, Heymann D, Harris M. National UK programme of community health workers for covid-19 response. Lancet. 2020;395(10231):1173-5. https://doi.org/10.1016/S0140-6736(20)30735-2.

24. Daumas RP, Silva GA, Tasca R, Leite IC, Brasil P, Greco DB, et al. The role of primary care in the Brazilian healthcare system: limits and possibilities for fighting COVID-19. Cad Saude Publica. 2020;36(6):e00104120. https://doi.org/10.1590/0102-311X00104120.

25. Ministério da Saúde (BR). Ofício n. 246, de 15 maio de 2020. Resultado parcial da campanha nacional de vacinação contra influenza: 2020. Brasília, DF: MS; 2020 [citado 11 jan 2021]. Disponível em:

https://sei.saude.gov.br/sei/controlador_externo.php?acao=documento_conferir\&id_orga o_acesso_externo=0

26. Savassi LCM, Bedetti AD, Abreu ABJ, Costa AC, Perdigão RMC, Ferreira TP. Ensaio acerca das curvas de sobrecarga da covid-19 sobre a atenção primária. J Manag Prim Health Care. 2020;12:e38. https://doi.org/10.14295/jmphc.v12.1006.

27. Turci MA, Holliday JB, Oliveira NCVC. A vigilância epidemiológica diante do Sars-Cov-2: desafios para o SUS e a atenção primária à saúde. APS Rev. 2020;2(1):44-55. https://doi.org/10.14295/aps.v2i1.70.

28. Ministério da Saúde (BR). Recomendações para os profissionais de saúde no âmbito das equipes de referência para a população do campo, floresta e águas referentes ao covid19. Brasília, DF: Secretaria de Atenção Primária à Saúde; c2020 [citado 25 out 2020]. Disponível em: http://fi-admin.bvsalud.org/document/view/5ctvc 
Átila Augusto Cordeiro Pereira | https://orcid.org/0000-0002-6452-5493 http://lattes.cnpq.br/ 3520714316934552

Diully Siqueira Monteiro | https://orcid.org/0000-0002-9974-061X http://lattes.cnpq.br/5607026180861004

Sâmela Stefane Correa Galvão | https://orcid.org/0000-0003-1107-960X http://lattes.cnpq.br/4246605452546557

Layse Viana Figueiredo Garcia | https://orcid.org/0000-0003-2418-1195 http://lattes.cnpq.br/4443504565130701

Tamilis Feitosa Leal | https://orcid.org/0000-0001-5934-2222 http://lattes.cnpq.br/3102391864642290

João Victor Moura Rosa| https://orcid.org/0000-0002-8849-7730 http://lattes.cnpq.br/4313376872732929

Sâmia Cristine Rabelo Borges | https://orcid.org/0000-0002-9163-5188 http://lattes.cnpq.br/6849084861392422 